\title{
A PARTICIPAÇÃO DO PAI NO PROCESSO DE AMAMENTAÇÃO
}

\author{
Janete Pereira Limaํㅡ, Luiza Helena de Oliveira Cazola², Renata Palópoli Pícoli
}

RESUMO: Este estudo objetivou identificar a participação do pai no processo de amamentação em uma maternidade estadual da região centro-oeste do Brasil. Pesquisa descritiva de abordagem quantitativa, utilizandose de dados primários coletados por meio de entrevistas estruturadas, junto a 56 pais acompanhantes de suas puérperas. Predominaram pais na faixa etária de 25 a 31 anos, 20 (35,71\%), casados, 22 (39,29\%) e com ensino médio completo, $18(32,14 \%)$. Todas as gestantes realizaram pré-natal e $36(64,29 \%)$ pais as acompanharam. Receberam orientações sobre amamentação, $23(41,07 \%)$ pais e dentre os profissionais que forneceram informações, predominou o enfermeiro $17(30,36 \%)$. O principal fator facilitador para os pais ajudarem na amamentação foi a vontade de apoiar a esposa $37(66,08 \%)$ e o dificultador, conciliar o horário de trabalho, 19 (33,93\%). Estar junto da mulher é a maneira que os pais encontraram para favorecer a amamentação e sua participação é fundamental para o sucesso desse processo.

DESCRITORES: Aleitamento materno; Cuidado pré-natal; Gestantes; Paternidade.

\section{INVOLVEMENT OF FATHERS IN THE BREASTFEEDING PROCESS}

\begin{abstract}
The aim of the present study was to identify the involvement of fathers in the breastfeeding process in a state maternity hospital in the Central-West of Brazil. A descriptive study with a quantitative approach was carried out with the use of primary data collected by means of structured interviews, with 56 fathers accompanying their puerperal partners. There was a prevalence of fathers aged from 25 to 31 years, $20(35.71 \%)$, married, $22(39.29 \%)$, and with complete high school education, 18 (32.14\%). All pregnant women carried out prenatal appointments and $36(64.29 \%)$ fathers accompanied them. Twenty-three (41.07\%) fathers received guidance on breastfeeding and there was a prevalence of nurses, 17 (30.36\%), among the professionals who provided them with information. The main facilitating factor for fathers to help in breastfeeding was the desire to support their wives, 37 (66.08\%), and the main difficult factor was the adjustment of working hours, $19(33.93 \%)$. Being next to their partners is the way fathers found to encourage breastfeeding, and their involvement is fundamental to the success of this process.
\end{abstract}

DESCRIPTORS: Breast Feeding; Prenatal Care; Pregnant Women; Paternity.

\section{PARTICIPACIÓN DEL PADRE EN EL PROCESO DE AMAMANTAMIENTO}

RESUMEN: Este estudio buscó identificar la participación del padre en el proceso de amamantamiento, en maternidad estatal de región centro-oeste de Brasil. Investigación descriptiva de abordaje cuantitativo, utilizando datos primarios recogidos mediante entrevistas estructuradas realizadas a 56 padres acompañantes de sus puérperas. Predominaron padres en faja etaria de 25 a 31 años, $20(35,71 \%)$ casados, $22(39,29 \%)$ y con enseñanza media completa, $18(32,14 \%)$. Todas las parturientas realizaron prenatal y $36(64,29 \%)$ padres las acompañaron. Recibieron orientación sobre amamantamiento 23 (41,07\%) padres. Entre los profesionales que brindaron información predominó el enfermero, $17(30,36 \%)$. El principal factor facilitador para que los padres ayuden en el amamantamiento fue la voluntad de apoyar a la esposa, 37 (66,08\%), y el dificultador, conciliar los horarios laborales, 19 (33,93\%). Permanecer junto a la mujer es el modo que los padres encontraron para facilitar el amamantamiento, su participación es fundamental para el éxito del proceso.

DESCRIPTORES: Lactancia Materna; Atención Prenatal; Mujeres Embarazadas; Paternidad.

${ }^{1}$ Enfermeira. Pós-graduanda em Enfermagem Obstétrica. Universidade Federal de Mato Grosso do Sul. Campo Grande, MS, Brasil.

Enfermeira. Doutora em Saúde e Desenvolvimento. Docente do Programa de Pós-Graduação do Mestrado Profissional em Saúde da Família da Universidade Federal de Mato Grosso do Sul. Campo Grande, MS, Brasil.

${ }^{3}$ Fonoaudióloga. Doutora em Saúde Pública. Pesquisadora da Fundação Oswaldo Cruz de Mato Grosso do Sul. Campo Grande, MS, Brasil.

Autor Correspondente:

Recebido: $27 / 07 / 2016$

Luiza Helena de Oliveira Cazola

Universidade Federal de Mato Grosso do Sul

Finalizado: 03/02/2017

R. Coronel Cacildo Arantes, 365 - 79040452 - Campo Grande, MS, Brasil

E-mail - luizacazola@gmail.com 


\section{INTRODUÇÃO}

O Aleitamento Materno (AM) é o modo de alimentação mais antigo e efetivo para a espécie humana. A influência desse alimento na saúde da criança é de suma importância, sendo apontado como o modo mais apropriado para o desenvolvimento saudável dos lactentes e o único alimento eficaz em atender adequadamente todas as necessidades fisiológicas das crianças menores de seis meses ${ }^{(1)}$.

A recomendação para que a criança receba o leite materno nos seis primeiros meses de vida de forma exclusiva e até os dois anos como complemento, possui bases científicas. Várias são as vantagens do AM: redução de casos de diarreia, pneumonia, ganho de peso, além de não ter risco de contaminação, ser barato e estar sempre pronto ${ }^{(2)}$.

O envolvimento paterno na amamentação, nos primeiros 10 dias após o parto, é de extrema importância para que haja continuidade do aleitamento materno, devido às dificuldades que habitualmente podem ocorrer na amamentação. É fundamental que se forme um elo entre mãepai-bebê desde a gestação. A presença mais ativa do pai na fase de preparação para a maternidade encorajaria a mãe a amamentar por mais tempo, a aprovação do pai para a amamentação é um fator primordial para o sucesso do $\mathrm{AM}^{(3)}$.

O conhecimento dos pais quanto aos benefícios da ama $\neg$ mentação, assim como seu apoio, compreensão e suporte na tomada de decisões juntamente com as mães podem ser itens relevantes na hora em que elas oferecem o leite materno aos filhos ${ }^{(4)}$.

Embora a participação do homem no processo de amamentação esteja ganhando notória importância, ainda é pequeno o que se sabe sobre seus sentimentos no processo de aleitamento materno de seu filho e quais suas dificuldades e demandas para que possa ajudar mais e vivenciar de forma positiva esse momento ${ }^{(5)}$.

Assim, diante da relevância da participação do pai nesse processo, este estudo teve por objetivo identificar a sua participação no processo de amamentação, junto às puérperas atendidas em um Hospital Amigo da Criança, na cidade de Campo Grande, no estado de Mato Grosso do Sul.

\section{- MÉTODO}

Esse estudo é descritivo, exploratório, conduzido pela abordagem quantitativa, realizado em uma Maternidade Escola em Campo Grande, Mato Grosso do Sul, Brasil. A unidade hospitalar recebeu a distinção de "Amigo da Criança", considerada referência para assistência materno-infantil pelo Ministério da Saúde.

Os participantes foram o universo de 56 homens, pais dos bebês nascidos na Maternidade pesquisada, acompanhantes de suas puérperas. Essa quantidade foi definida por meio da identificação dos pais no registro do instrumento denominado Censo Diário, que permitiu verificar a presença dos mesmos como acompanhantes, durante o período de coleta dados, que obedeceram aos seguintes critérios de inclusão: pais que estiveram presentes na maternidade no período puerperal e com seus recém-nascidos em amamentação exclusiva ao seio materno. Foram excluídos pais menores de 18 anos de idade, indígenas, quilombolas e os que suas puérperas receberam alta antes que se pudesse realizar a entrevista.

Para a coleta de dados, utilizou-se entrevista estruturada aplicada junto aos pais pela própria pesquisadora, em local reservado na própria maternidade, no período de 17 de dezembro de 2015 a 31 de janeiro de 2016, guiada por um roteiro cujas perguntas abordaram as seguintes variáveis: características sociodemográficas, acompanhamento pré-natal, orientações quanto à amamentação, aspectos facilitadores e dificultadores da participação do pai no processo de amamentar e atitudes do pai que favorecem a amamentação.

Os resultados foram apresentados em forma de tabelas e analisados pela estatística descritiva, com frequência absoluta e relativa, discutidos a partir da literatura pertinente. 
O projeto de pesquisa foi aprovado pelo Comitê de Ética em Pesquisa de Seres Humanos da Universidade Federal de Mato Grosso do Sul, sob parecer $\mathrm{n}^{\circ}$ 1.371.269.

\section{RESULTADOS}

A Tabela 1 apresenta as características sociodemográficas da população do estudo. Predominou a faixa etária de 25 a 31 anos 20 (35,71\%) e o estado civil casado 22 (39,29\%), seguido de união estável $21(37,50 \%)$. Quanto à variável escolaridade, o ensino médio completo 18 (32,14\%) e o ensino médio incompleto $15(26,79 \%)$ foram os de maior percentual.

Tabela 1 - Características sociodemográficas dos pais. Campo Grande, MS, Brasil, 2016

\begin{tabular}{lcc} 
Variáveis & $\mathbf{n}$ & $\%$ \\
\hline Idade (anos) & & \\
\hline 18 a 24 & 19 & 33,93 \\
\hline 25 a 31 & 20 & 35,71 \\
\hline 32 a 38 & 9 & 16,07 \\
\hline 39 a 46 & 8 & 14,29 \\
\hline Estado civil & & \\
\hline Casado & 22 & 39,29 \\
\hline União Estável & & 37,5 \\
\hline Solteiro & 13 & 23,21 \\
\hline Escolaridade & & \\
\hline Sem escolaridade & 1 & 1,79 \\
\hline Ensino Fundamental Completo & 6 & 10,71 \\
\hline Ensino Fundamental Incompleto & 10 & 17,85 \\
\hline Ensino Médio Completo & 18 & 32,14 \\
\hline Ensino Médio Incompleto & 15 & 26,79 \\
\hline Nível Superior Completo & 1 & 1,79 \\
\hline Nível Superior Incompleto & 5 & 8,93
\end{tabular}

A Tabela 2 demonstra que, na sua totalidade, 56 (100\%) das puérperas realizaram o pré-natal e que a maioria $46(82,14 \%)$, buscou atendimento na rede de serviço público, e foram acompanhadas para o atendimento do pré-natal por mais da metade de seus companheiros $36(64,29 \%)$. Com relação às orientações prestadas sobre amamentação, mais da metade dos pais $33(58,93 \%)$ não recebeu nenhuma orientação e dentre os que receberam 23 (41,07\%), as avaliaram em sua maioria como ótimas $17(73,21 \%)$. O profissional que mais prestou orientações foi a enfermeira 17 (30,36\%), seguida dos profissionais médicos pediatras 13 (23,21\%) e obstetras 10 (17,86\%).

Tabela 2 - Distribuição de puérperas que realizaram acompanhamento do pré-natal e orientações recebidas pelo companheiro sobre amamentação. Campo Grande, MS, Brasil, 2016 (continua)

\begin{tabular}{lcc} 
Variáveis & n & \% \\
\hline Pré-natal & & \\
\hline Sim & 56 & 100 \\
\hline Rede de serviço & & \\
\hline Público & 46 & 82,14 \\
\hline Privado & 10 & 17,86 \\
\hline
\end{tabular}




\begin{tabular}{lcc}
\hline Acompanhamento do pré-natal pelo companheiro & & \\
\hline Sim & 36 & 64,29 \\
\hline Não & 20 & 35,71 \\
\hline Recebeu orientação & 33 & 58,93 \\
\hline Não & 23 & 41,07 \\
\hline Sim & & \\
\hline Avaliação da orientação & 17 & 73,91 \\
\hline Ótima & 4 & 17,4 \\
\hline Boa & 2 & 8,69 \\
\hline Regular & 0 & 0 \\
\hline Péssima & & \\
\hline Profissional que prestou a orientação* & 17 & 30,36 \\
\hline Enfermeira & 13 & 23,21 \\
\hline Médico pediatra & 10 & 17,86 \\
\hline Médico obstetra & 6 & 10,71 \\
\hline Profissional do banco de leite & 5 & 8,93 \\
\hline Técnico de enfermagem & 3 & 5,36 \\
\hline Outros & 2 & 3,57 \\
\hline Acadêmico de enfermagem & &
\end{tabular}

*Os pais puderam opinar por mais de uma resposta

Quanto aos aspectos referidos pelos pais que facilitaram sua participação na amamentação, o apoio à esposa foi considerado como o principal $37(66,08 \%)$ e como maior dificuldade referiram ter de conciliar o horário de trabalho 19 (33,93\%), seguido pelo problema de manter-se acordado à noite 11 (19,64\%). Como atitudes favorecedoras da amamentação, predominaram poder estar junto da companheira durante o processo de amamentação 31 (55,37\%) e ajudá-la a posicionar o recém-nato em seus braços $28(50,02 \%)$, como mostra a Tabela 3.

Tabela 3 - Aspectos facilitadores e dificultadores da participação do pai e suas atitudes favorecedoras para a amamentação. Campo Grande, MS, Brasil, 2016

\begin{tabular}{lcc} 
Variáveis & $\mathbf{n}$ & $\mathbf{\%}$ \\
\hline Facilitadores* & & \\
\hline Apoio à esposa & 37 & 66,08 \\
\hline Envolvimento com a mãe e o filho & 18 & 32,15 \\
\hline Conhecimento do assunto & 7 & 12,5 \\
\hline Ter mais tempo livre & 4 & 7,15 \\
\hline Dificultadores* & \\
\hline Conciliar o horário do trabalho & 19 & 33,93 \\
\hline Manter-se acordado à noite & 11 & 19,64 \\
\hline Falta de informação & 9 & 16,07 \\
\hline Medo & 1 & 1,79 \\
\hline Não referiu dificuldades & 16 & 28,57 \\
\hline Atitudes favorecedoras* & & \\
\hline Estar junto & 31 & 55,37 \\
\hline Posicioná-lo nos braços da mãe & 28 & 50,02 \\
\hline Encorajar a mãe a amamentar & 22 & 39,29 \\
\hline Pegar no berço & 16 & 28,59 \\
\hline Outros & 2 & 3,57
\end{tabular}




\section{- DISCUSSÃO}

Os resultados dessa pesquisa revelaram maiores percentuais de pais na faixa etária de 25 a 31 anos, semelhantes a estudo realizado no Hospital das Clínicas de Uberlândia (MG), cuja faixa etária predominou entre 26 a 39 anos, o que sugere que pais adultos jovens possuem maior conhecimento sobre aleitamento materno ${ }^{(6)}$.

A presença de companheiro fixo contribui para que as mães prolonguem o seu período de lactação(7). Constatou-se nesse estudo que essa característica deve ser considerada como um elemento positivo, pois a maioria dos pais se declarou casado, seguido de união estável.

Com relação à escolaridade, o ensino médio completo e o incompleto foram os que apresentaram percentuais mais elevados, achados semelhantes a um estudo realizado no Hospital Regional da Região Administrativa de Ceilândia (DF) ${ }^{(8)}$. Maior grau de escolaridade dos pais oportuniza a busca de informações que possam auxiliar suas companheiras e filhos no processo de amamentação(6).

Buscando ampliar o acesso e a melhoria da qualidade do pré-natal, a implementação de boas práticas na atenção ao parto e nascimento, incluindo o direito ao acompanhante de livre escolha da mulher no parto, é lançada em 2011 a Rede Cegonha ${ }^{(9)}$.

Essa ampliação de acesso pode ter contribuído para que a totalidade das gestantes desse estudo realizasse consultas de pré-natal, corroborando com pesquisa realizada em Pelotas (RS) cujo resultado foi de $97,90 \%{ }^{(10)}$. A percepção das gestantes quanto ao pré-natal é visto como um cuidado fundamental nesse período, principalmente, para a saúde da criança e para o cuidado com a sua própria saúde ${ }^{(11)}$.

Em uma pesquisa qualitativa envolvendo puérperas, todas relataram terem sido atendidas em um hospital privado ${ }^{(12)}$, contrariando esse estudo, que identificou predomínio de busca por atendimentos nos serviços da rede pública.

Uma estratégia adotada pelo governo federal foi a implantação do chamado pré-natal do parceiro, cuja intenção é dar oportunidade para que os homens quando forem com suas companheiras às consultas de pré-natal, possam também cuidar de sua saúde, cujo propósito é gerar vínculos afetivos saudáveis e qualidade de vida para todos da famíliaa ${ }^{(13)}$.

Questionados quanto ao acompanhamento paterno durante a consulta de pré-natal, 36 (64,29\%) responderam que suas parceiras foram por eles acompanhadas, atitude essa que demonstra importante mudança no comportamento dos pais. Situação oposta foi encontrada em uma pesquisa realizada no município de Cáceres (MT) ${ }^{(14)}$, cujos índices chegaram a 23 (76\%) dos pais, ou seja, mais da metade não acompanhou as consultas de pré-natal.

Em estudo realizado no município de Dourados (MS), a totalidade dos pais relatou estarem satisfeitos em poderem acompanhar suas parceiras nas consultas de pré-natal, o que possibilita um maior conhecimento das mudanças na gestação, diminuindo suas angústias, resultantes de dúvidas sobre os cuidados com seu filho(15).

A inserção do pai nas atividades educativas durante o pré-natal e puerpério são fundamentais, já que os cuidados com o bebê é tarefa do casal. Quando o pai é estimulado e orientado a cuidar de seu filho, ajudará a mãe a vencer os obstáculos que poderão aparecer no processo de amamentação ${ }^{(16)}$. Nesse estudo, identificou-se que mais da metade dos pais não recebeu orientações sobre amamentação. Contudo, outra pesquisa identificou que apenas um terço (34,7\%) não recebeu orientações ${ }^{(4)}$.

Em 2004, o Ministério da Saúde lançou a "Política Nacional de Atenção Integral à Saúde da Mulher" (PNAISM), que tem como proposta incentivar a participação da enfermagem nas ações de saúde da mulher, especialmente no pré-natal, dentre elas ações educativas, que colocam a mulher como sujeito ativo no cuidado de sua saúde ${ }^{(17)}$.

O profissional que mais prestou orientações aos pais foi a enfermeira, seguida dos médicos pediatra e obstetra, sendo essas avaliadas como ótimas. As instituições de saúde devem preparar seus profissionais envolvidos com o processo de amamentação, para que possam melhorar a qualidade da assistência prestada de maneira a apoiar e acolher o casal ${ }^{(18)}$. 
Diante dos aspectos facilitadores da participação do pai na amamentação, o apoio à esposa foi apontado como o principal. Outra pesquisa revelou que os pais se revelaram participativos nessa fase, atuando diretamente em situações favoráveis ao ato de aleitar ${ }^{(3)}$. Por outro lado, conciliar o horário de trabalho e manter-se acordado à noite foram apontados como os principais dificultadores, achados semelhantes aos relatados pelos pais em um estudo realizado na cidade de Bauru-SP(19).

Como estratégia de ampliação e incentivo para a participação do pai nesse processo, criou-se a Política Nacional Integrada para a Primeira Infância, alterando a extensão da licença-paternidade de cinco para vinte dias. Ao assegurar a participação do pai nos primeiros momentos de vida dos filhos, a presença paterna torna-se um direito da criança e viabiliza uma melhor divisão entre homens e mulheres nas tarefas de cuidados com as crianças $^{(20)}$.

Constatou-se que estar junto da companheira e posicionar a criança nos braços da mãe, são atitudes que os pais entrevistados encontraram para favorecer a amamentação. Atitudes semelhantes foram identificadas em um estudo realizado com pais usuários do Sistema Único de Saúde da região Leste de Goiânia, cujos pais relataram disposição para ajudar a parceira no período da amamentação, acalentando a criança quando chorosa e colocando-a no colo da mãe para amamentar ${ }^{(21)}$.

\section{CONCLUSÕES}

O presente estudo evidenciou predomínio dos pais na faixa etária entre 25 - 31 anos, casados e com ensino médio completo. Todas as gestantes realizaram pré-natal e na sua maioria foram acompanhadas por seu parceiro, atitude essa que irá favorecer a participação do pai no processo de amamentação.

A quantidade de pais que não receberam orientações sobre amamentação é preocupante e, quando recebidas, a enfermeira foi o profissional mais mencionado. A participação dos profissionais responsáveis pelo pré-natal é fundamental nesse processo de orientação e inclusão paterna. Pode-se notar que ainda são necessários mais investimentos em ações educativas, e que o pai seja levado e encorajado a participar mais desse processo.

Estar junto da esposa é a maneira que os pais encontraram para favorecer a amamentação, de modo que sua participação é fundamental para o sucesso desse processo.

Este estudo apresentou limitações, pelo fato de se conseguir identificar apenas os pais cujos nomes constavam no registro do instrumento denominado Censo Diário. Recomenda-se a unidade de saúde, portanto, um maior rigor no preenchimento dos dados, o que irá favorecer estudos futuros.

\section{REFERÊNCIAS}

1. Pereira GOM. Educação em saúde no pré-natal para o fortalecimento do aleitamento materno [monografia]. Fortaleza (CE): Escola de Saúde Pública do Ceará; 2010.

2. Salvador JP, Ximenes VL, da Silva ICM, da Silva MFP. Participação do companheiro na promoção do aleitamento materno exclusivo em hospital amigo da criança. Revista interdisciplinar NOVAFAPI. 2012;5(1):30-6.

3. Piazzalunga CRC, Lamounier JA. O contexto atual do pai na amamentação: uma abordagem qualitativa. Rev. Med. Minas Gerais. 2011;21(2):133-41.

4. da Silva PP, Silveira RB, Mascarenhas MLW, Silva MB, Kaufmann CC, Albernaz EP. A percepção das mães sobre o apoio paterno: influência na duração do aleitamento materno. Rev. paul. pediatr. 2012;30(3):306-13.

5. Zutin TLM. A Posição do homem no processo de amamentação: um ensaio sobre a produção de sentimentos [tese]. São Paulo (SP): Escola de Enfermagem da Universidade de São Paulo; 2012.

6. de Resende TC, Dias EP, Cunha CMC, de Mendonça GS, Ribeiro Júnior AL, Santos LRL, et al. Participação paterna no período da amamentação: importância e contribuição. Biosci. J. 2014;30(3):925-32.

7. Rocci E, Fernandes RAQ. Dificuldades no aleitamento materno e influência no desmame precoce. Rev. bras. 
enferm. 2014;67(1):22-7.

8. Campanati FLS. Participação paterna no ciclo gravídico puerperal: vivências e sentimentos [monografia]. Brasília (DF): Universidade Federal de Brasília; 2015.

9. Ministério da Saúde (BR). Portaria n. 1459, de 24 de Junho de 2011. Institui no âmbito do Sistema Único de Saúde (SUS) a Rede Cegonha. Diário oficial da União, 24 jun 2011.

10. da Rosa SV, Torres AAP, Soares MC, Kerber NPC. Apoio à amamentação às adolescentes em um hospital de ensino do sul do Brasil. J. Nurs Health. 2013;3(1):16-26.

11. Barreto CN, Ressel LB, dos Santos CC, Wilhelm LA, da Silva SC, Alves CN. Atenção pré-natal na voz das gestantes. Rev enferm UFPE on line. [Internet] 2013;7(5) [acesso em 13 mar 2016]. Disponível: http://www.revista. ufpe.br/revistaenfermagem/index.php/revista/article/download/4355/6376.

12. Francisquini AR, Higarashi IH, Serafim D, Bercini LO. Orientações recebidas durante a gestação, parto e pósparto por um grupo de puérperas. Cienc Cuid Saude. 2010;9(4):743-51.

13. Brasil. Portal Brasil. Dilma sanciona marco legal da primeira infância. [Internet] 2014 [acesso em 13 mar 2016]. Disponível: http://www.brasil.gov.br/governo/2014/04/dilma-sanciona-o-marco-civil-da-internet.

14. Ferreira TN, de Almeida DR, de Brito HM, Cabral JF, Marin HA, Campos FMC, et al. A Importância da participação paterna durante o pré-natal: percepção da gestante e do pai no município de Cáceres-MT. G\&S. 2014;5(2):337-45.

15. de Figueiredo MGAV, Marques AC. Pré-natal: experiências vivenciadas pelo pai. Cogitare Enferm. 2011;16(4):708-13.

16. da Cruz DSM, Rocha INSBS, Marques DKA, de Souza IVB. Percepção da figura paterna frente ao aleitamento materno. Cogitare Enferm. 2011;16(4):702-7.

17. Ministério da Saúde (BR). Secretaria de Atenção à Saúde. Política Nacional de Atenção Integral à Saúde da Mulher: princípios e diretrizes. Brasília: Ministério da Saúde; 2011.

18. Cabral PP, Barros CS, de Vasconcelos MGL, Javorski M, Pontes CM. Motivos do sucesso da amamentação exclusiva na perspectiva dos pais. Rev. Eletr. Enf. [Internet] 2013;15(2) [acesso em 20 mar 2016]. Disponível: http:// dx.doi.org/10.5216/ree.v15i2.16996.

19. Pinho AMS. Compreendendo a vivência do pai frente à amamentação [dissertação]. São Paulo (SP): Universidade Estadual Paulista Júlio de Mesquita Filho, Faculdade de Medicina de Botucatu; 2012.

20. Ministério da Saúde (BR). Pré-natal do parceiro incentiva homens a cuidarem da saúde. [Internet] 2014 [acesso em 10 jan 2016]. Disponível: http://portalsaude.saude.gov.br/index.php/cidadao/principal/agencia-saude/15674pre-natal-do-parceiro-incentiva-homens-a-cuidarem-da-saude.

21. Paula AO, Sartori AL, Martins CA. Aleitamento Materno: orientações, conhecimento e participação do pai nesse processo. Rev. Eletr. Enf. [Internet] 2010;12(3) [acesso em 10 jan 2016]. Disponível: http://dx.doi.org/10.5216/ ree.v12i3.6929. 